\title{
Lung abscess without sepsis in a patient with diabetes with refractory episodes of spontaneous hypoglycemia: a case report and review of the literature
}

\author{
Gotaro Toda, Midori Fujishiro", Tomohide Yamada, Nobuhiro Shojima, Hideyuki Sakoda, Ryo Suzuki, \\ Toshimasa Yamauchi, Kohjiro Ueki and Takashi Kadowaki
}

\begin{abstract}
Introduction: Hypoglycemia is a cause of considerable morbidity. Although hypoglycemia has been documented in the setting of septic shock and has been associated with higher mortality, hypoglycemia in infection without sepsis has not been reported in the literature.

Case presentation: A 72-year-old Japanese woman treated with high-dose glucocorticoids for autoimmune hemolytic anemia, as well as intensive insulin therapy for type 2 diabetes, presented with severe hypoglycemia. A lung abscess was diagnosed by imaging studies and treated with intravenous antibiotics. Hypoglycemia spontaneously recurred during lung abscess exacerbations, despite appropriate de-escalation of antidiabetic therapy. Only mild sporadic episodes of hypoglycemia occurred after the lung abscess was controlled. Infection accompanied with malnutrition and immunosuppression, although in the absence of sepsis, may have contributed to hypoglycemia.

Conclusions: Caution is warranted in the management of hypoglycemia in patients with diabetes with the conditions described here, that is malnutrition and immunosuppression, as infection may be a contributing factor.
\end{abstract}

Keywords: Hypoglycemia, Type 2 diabetes, Infection, Insulin resistance, Glucocorticoids

\section{Introduction}

Hypoglycemia is a cause of considerable morbidity, and is of major concern throughout the treatment of diabetes mellitus. Although infection is known to exacerbate insulin resistance and cause hyperglycemia, septic shock produces hypoglycemia. However, to the best of our knowledge, hypoglycemia in infection without sepsis has not been reported in the literature. Here, we present the case of a patient experiencing refractory episodes of spontaneous hypoglycemia in which lung abscess, although without sepsis, may have played a central role.

\section{Case presentation}

A 72-year-old Japanese woman presented to a neighboring hospital with loss of consciousness due to severe

* Correspondence: midori-tky@umin.ac.jp

Department of Diabetes and Metabolic Diseases, Graduate School of Medicine, The University of Tokyo, 7-3-1, Hongo, Bunkyo-ku, Tokyo, Japan hypoglycemia (plasma glucose $10 \mathrm{mg} / \mathrm{dl}$ ). She was transferred to our facility the following day. She had had type 2 diabetes, treated with insulin (basal-supported oral therapy) for three years before admission. Three months before admission, she was diagnosed with autoimmune hemolytic anemia (AIHA) and was started on $70 \mathrm{mg}$ oral prednisolone per day, which exacerbated her diabetes, necessitating intensive insulin therapy. By two weeks before admission, oral prednisolone was tapered to $50 \mathrm{mg}$ as her hemoglobin concentration had stabilized around $10 \mathrm{~g} / \mathrm{dl}$. She experienced no episodes of overt hypoglycemia prior to hospitalization.

Our patient was obese with a body mass index of $31.2 \mathrm{~kg} / \mathrm{m}^{2}$. Her medications included insulin (27 units/day), and oral prednisolone $(50 \mathrm{mg} /$ day). Her insulin dosage was 7 units of insulin aspart before breakfast, 4 units before other meals, and 12 units of insulin glargine at bedtime. Chest radiography on admission revealed a high-density 

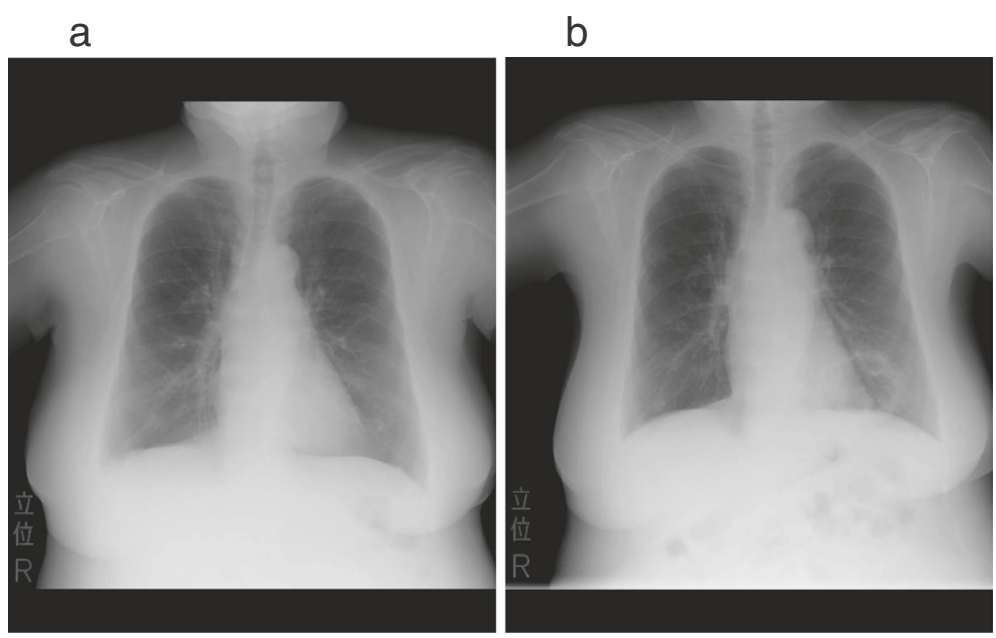

Figure 1 Chest radiographs obtained one month before admission (a), and on admission (b). A large hollow lesion in the lower left lung field was diagnosed as a lung abscess.

area with a hollow lesion not detected the previous month (Figure 1), which was confirmed by computed tomography. A lung abscess was diagnosed and intravenous $4.5 \mathrm{~g}$ piperacillin/tazobactam every 8 hours was started. Her sputum and blood culture test results were negative. Laboratory examinations showed elevated serum C-reactive protein $(7.32 \mathrm{mg} / \mathrm{dl})$, and normal fasting adrenocorticotropic hormone $(\mathrm{ACTH})(19.1 \mathrm{pg} / \mathrm{ml})$ and cortisol $(10.6 \mu \mathrm{g} / \mathrm{ml})$ levels despite administration of $50 \mathrm{mg}$ of prednisolone per day. Her fasting and postprandial plasma C-peptide levels were low (fasting and postprandial plasma glucose and C-peptide levels were $221 \mathrm{mg} / \mathrm{dl}$, $0.5 \mathrm{ng} / \mathrm{ml}$ and $293 \mathrm{mg} / \mathrm{dl}, 0.4 \mathrm{ng} / \mathrm{ml}$ respectively). Our patient's kidney function was normal with a serum creatinine level of $0.76 \mathrm{mg} / \mathrm{dl}$. The hemoglobin concentration remained stable $(10.5 \mathrm{~g} / \mathrm{dl})$, and hemoglobin $(\mathrm{Hb}) \mathrm{A} 1 \mathrm{c}$ was stable at $7.6 \%(59.6 \mathrm{mmol} / \mathrm{mol})$. Her serum albumin was low $(1.8 \mathrm{~g} / \mathrm{dl})$. Her anti-insulin antibody test was negative (Table 1).

Recurrent episodes of hypoglycemia continued after admission, and did not resolve until the 30th day of hospitalization, the point at which the lung lesion was largest (Figure 2, $8.7 \times 7.0 \mathrm{~cm}$ axially). Hypoglycemia was observed 16 times in the same period, with a maximum of three times in the same day (Figure 3). Episodes were observed before and after meals, and were not concentrated in a particular time of the day. After admission, her insulin dosage was reduced to 3 units of insulin aspart before meals, which was skipped when oral intake was insufficient. Her insulin glargine was not reduced, due to high levels of fasting plasma glucose. Oral therapy consisted of voglibose ( $0.2 \mathrm{mg}$ before meals), and buformine hydrochloride (100mg before breakfast). Buformine was discontinued shortly after admission. A dosage of $50 \mathrm{mg}$ oral prednisolone was constantly administered from
Table 1 Results of laboratory examinations on admission

\begin{tabular}{lll}
\hline White blood cells & 18,700 & $/ \mathrm{ul}$ \\
Band cells & 4.0 & $\%$ \\
Segmented cells & 93.0 & $\%$ \\
Eosinophils & 0.0 & $\%$ \\
Hemoglobin & 10.5 & $\mathrm{~g} / \mathrm{dl}$ \\
Platelet count & 294,000 & $\mathrm{/ul}$ \\
Serum albumin & 1.8 & $\mathrm{~g} / \mathrm{dl}$ \\
Serum lactate dehydrogenase & 432 & $\mathrm{U} / \mathrm{L}$ \\
Serum aspartate aminotransferase & 18 & $\mathrm{U} / \mathrm{L}$ \\
Serum alanine aminotransferase & 19 & $\mathrm{U} / \mathrm{L}$ \\
Serum blood urea nitrogen & 21.9 & $\mathrm{mg} / \mathrm{dl}$ \\
Serum creatinine & 0.76 & $\mathrm{mg} / \mathrm{dl}$ \\
Serum sodium & 136 & $\mathrm{mEq} / \mathrm{L}$ \\
Serum potassium & 4.7 & $\mathrm{mEq} / \mathrm{L}$ \\
Serum creatine kinase & 31 & $\mathrm{U} / \mathrm{L}$ \\
Serum glycoalbumin & 23.3 & $\%$ \\
Serum C-reactive protein & 7.32 & $\mathrm{mg} / \mathrm{dl}$ \\
Fasting adrenocorticotropic hormone & 19.1 & $\mathrm{pg} / \mathrm{ml}$ \\
Fasting cortisol & 10.6 & $\mathrm{ug} / \mathrm{ml}$ \\
HbA1c & 7.6 & $\mathrm{mg} / \mathrm{dl}$ \\
Fasting plasma glucose & 221 & $\mathrm{mg} / \mathrm{dl}$ \\
Fasting C-peptide reactivity & 0.5 & $\mathrm{ng} / \mathrm{ml}$ \\
Postprandial plasma glucose & 293 & \\
Postprandial C-peptide reactivity & 0.4 & $\mathrm{Negative}$ \\
Anti-insulin antibody & & \\
\hline HbA1c, hemoglobn A1c & & \\
\hline
\end{tabular}

HbA1c, hemoglobin A1c. 


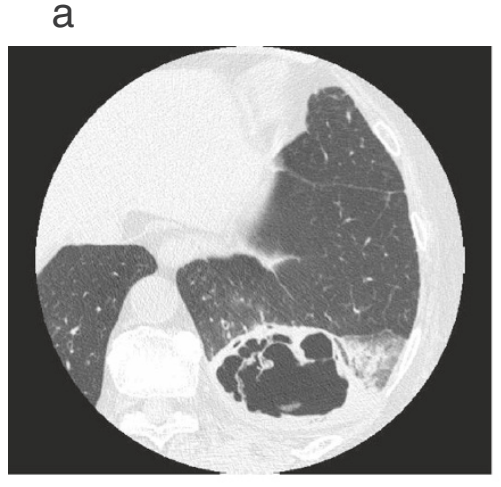

b

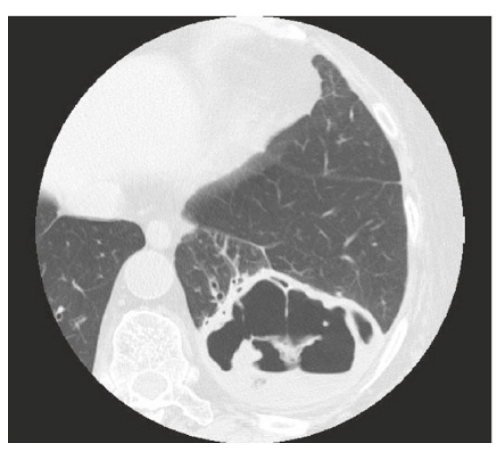

C

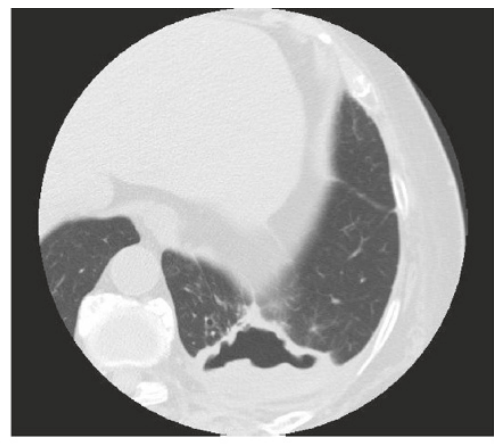

d

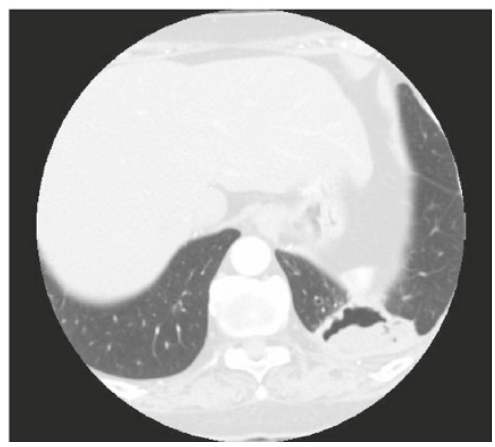

Figure 2 Computed tomographic images of the lung abscess on the second $(\mathrm{a}), 31^{\text {st }}(\mathrm{b}), 79^{\text {th }}(\mathrm{c})$, and $98^{\text {th }}(\mathrm{d})$ days after admission. The maximum diameters of the lesion were $60,87,60$, and $50 \mathrm{~mm}$, respectively. As shown here, the lesion was largest around the $30^{\text {th }}$ day after admission.

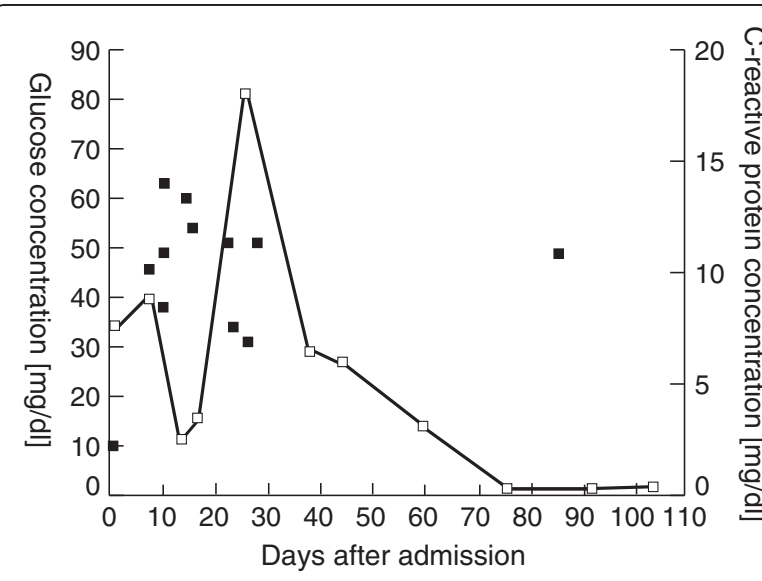

Figure 3 Hypoglycemic episodes after admission. Black squares represent glucose concentrations below $70 \mathrm{mg} / \mathrm{dl}$. Glucose concentrations were measured four or six times daily. White squares represent plasma C-reactive protein concentrations. Hypoglycemic episodes were mainly observed from admission to the $30^{\text {th }}$ day of hospitalization, during which the lung abscess was enlarging as shown in Figure 2. two weeks before admission until episodes of hypoglycemia ceased to appear. Episodes of hypoglycemia were not observed as the lesion decreased in size after this point (Figure 2, $5.0 \mathrm{~cm}$ axially on the $98^{\text {th }}$ day of hospitalization), and our patient was discharged from our hospital after two months of antibiotic treatment.

\section{Discussion}

Possible causative factors of recurrent hypoglycemia in the present case included insulin use, malnutrition, and severe infection resulting from the lung abscess. Insulin was unlikely to have played a central role, as no episodes of hypoglycemia were observed under administration of the same dosages of insulin before admission. Insulin dosages were appropriately skipped or tapered according to the amount of oral intake in unsuccessful attempts to avoid hypoglycemia during periods where hypoglycemia was observed. Oral intake was approximately $1000 \mathrm{kcal} /$ day, which was inadequate $(21 \mathrm{kcal}$ per ideal body weight). However, we speculate malnutrition was not central to the pathogenesis of hypoglycemia, as oral intake was similar before and after the episodes of hypoglycemia. The infection was likely to be severe and prolonged, as suggested by low serum albumin levels and a rise in $\mathrm{C}$-reactive protein to a maximum of 
$18.03 \mathrm{mg} / \mathrm{dl}$ on day 24 , though symptoms of infection in this patient may have been masked by glucocorticoid use. The normal levels of fasting ACTH and cortisol raised the possibility of very strong secretory stimuli, such as severe infection, and/or hypoglycemia causing a limited ACTH response even under high prednisolone doses. Moreover, the absence of hallmarks of adrenal insufficiency (stable systolic blood pressure at around $120 \mathrm{mmHg}$, normal levels of potassium and eosinophils throughout the patient's course) led us to believe adrenal insufficiency was not central in the pathogenesis of hypoglycemia. Supported by the observation that hypoglycemia and the lung abscess had exhibited parallel courses, severe infection may have played a central role in the pathogenesis of recurrent hypoglycemia in this patient.

Previous studies showed immunocompromised hosts with lung abscess to be more likely to develop lung abscesses with positive cultures for aerobes, as compared with immunocompetent hosts $(63 \%$ vs. $20 \% ; \mathrm{P}=0.057)$ [1], and that patients with lung abscess who have diabetes are at increased risk for lung abscess with positive cultures for Klebsiella pneumoniae compared to patients without diabetes (odds ratio 4.3, 95\% confidence interval, 1.0 to $18.4, P=0.039$ ) [2]. On these grounds, we speculate Gram-negative bacteria, such as $K$. pneumoniae, are candidates for the virulent organism in this patient receiving high-dose glucocorticoids in addition to having diabetes.

Hypoglycemia has been reported in $16.3 \%$ of patients with sepsis, and has been associated with higher mortality [3]. Extensive research has been conducted to elucidate the mechanisms of hypoglycemia in infection, using animal models injected with lipopolysaccharide (LPS). LPS induces hyperglycemia approximately 1 hour after injection into mice [4] followed by hypoglycemia within 6 hours [5]. Glycogen depletion, decreased gluconeogenesis, and increased glucose consumption have been suggested as mechanisms of hypoglycemia induction by LPS $[5,6]$. LPS inhibits insulin signaling in vivo, which leads to decreased glycogen synthesis, increased glycogenolysis and also increased gluconeogenesis [7]. Euglycemic hyperinsulinemic clamp studies on animal models of LPS-induced hyperglycemia support this view, revealing an increase in hepatic glucose output, possibly contributing to glycogen depletion [4]. LPS-induced cytokines such as interleukin (IL) $-1 \beta$ and tumor necrosis factor (TNF) $\alpha$ impair the glucagon-induced increase in gluconeogenesis in animal models [8]. LPS also downregulates cytoplasmic glucocorticoid receptors in the livers of mice, resulting in inhibition of the glucocorticoid-induced increase in hepatic gluconeogenesis [5]. Negative blood cultures on multiple occasions in the present case were not suggestive of sepsis. However, taking the above observations together, we speculate that hypoglycemia can be induced by severe prolonged infection without signs of sepsis in settings of malnutrition and immunosuppression, even with highdose glucocorticoid treatment, as in the present case.

\section{Conclusions}

Clinicians should exercise particular caution managing hypoglycemia in settings of malnutrition, immunosuppression, and glucocorticoid use, as severe bacterial infection may be involved in its pathogenesis.

\section{Consent}

Written informed consent was obtained from the patient for publication of this case report and any accompanying images. A copy of the written consent is available for review by the Editor-in-Chief of this journal.

\section{Competing interests}

The authors declare that they have no competing interests.

\section{Authors' contributions}

GT wrote the manuscript. GT, MF, TY, NS, HS, RS, TY, KU, and TK interpreted the patient data and contributed to the discussion. TK was the guarantor.

All authors read and approved the final manuscript.

Received: 6 April 2013 Accepted: 16 December 2013

Published: 13 February 2014

\section{References}

1. Mansharamani N, Balachandran D, Delaney D, Zibrak J, Silvestri R, Koziel H: Lung abscess in adults: clinical comparison of immunocompromised to non-immunocompromised patients. Respir Med 2002, 96:178-185.

2. Wang J, Chen K, Fang C, Hsueh P, Yang P, Chang S: Changing bacteriology of adult community-acquired lung abscess in Taiwan: Klebsiella pneumoniae versus anaerobes. Clin Infect Dis 2005, 40:915-922.

3. Ssekitoleko R, Jacob S, Banura P, Pinkerton R, Meya D, Reynolds S: Hypoglycemia at admission is associated with inhospital mortality in Ugandan patients with severe sepsis. Crit Care Med 2011, 39:2271-2276.

4. Sugita H, Kaneki M, Tokunaga E, Sugita M, Koike C, Yasuhara S, Tompkins R, Jeevendra Martyn J: Inducible nitric oxide synthase plays a role in LPS-induced hyperglycemia and insulin resistance. Am J Physiol Endocrinol Metab 2002, 282:E386-E394.

5. Stith R, McCallum R: Down regulation of hepatic glucocorticoid receptors after endotoxin treatment. Infect Immun 1983, 40:613-621.

6. Oguri S, Motegi K, Yoichiro I, Yasuo E: Primary role of interleukin-1a and interleukin-1 $\beta$ in lipopolysaccharide-induced hypoglycemia in mice. Clin Diagn Lab Immunol 2002, 9:1307-1312.

7. Ueki K, Kondo T, Kahn R: Suppressor of cytokine signaling 1 (SOCS-1) and SOCS-3 cause insulin resistance through inhibition of tyrosine phosphorylation of insulin receptor substrate proteins by discrete mechanisms. Mol Cell Biol 2004, 24:5434-5446.

8. Christ B, Nath A: Impairment by interleukin $1 \beta$ and tumour necrosis factor a of the glucagon-induced increase in phosphoenolpyruvatecarboxykinase gene expression and gluconeogenesis in cultured rat hepatocytes. Biochem J 1996, 320:161-166.

doi:10.1186/1752-1947-8-51

Cite this article as: Toda et al:: Lung abscess without sepsis in a patient with diabetes with refractory episodes of spontaneous hypoglycemia: a case report and review of the literature. Journal of Medical Case Reports 2014 8:51. 